\title{
Rabdomiólisis asociada a electroestimulación muscular
}

\author{
Rhabdomyolisis related to electromyostimulation
}

La rabdomiólisis es un síndrome clínico caracterizado por la destrucción del músculo estriado y la liberación al plasma del contenido de sus células. Las causas más comunes son traumatismos, ejercicio físico, infecciones, fármacos, alteraciones endocrinológicas y miopatías primarias (1). Su espectro de gravedad es amplio, variando desde elevaciones asintomáticas de la creatincinasa (CK) hasta cuadros de mioglobinuria con insuficiencia renal aguda o hiperpotasemia graves (2). Presentamos un caso de rabdomiólisis asociada a la utilización de un dispositivo de electroestimulación muscular (EESM).

Mujer de 25 años sin antecedentes personales o familiares de interés, practicante habitual de ejercicio aeróbico. Consultó por cuadro de mialgias y debilidad en extremidades inferiores tras haberse sometido a una única sesión de EESM durante 20 minutos. La exploración mostró tumefacción en ambos muslos con dolor local a la palpación. En las exploraciones complementarias destacaban CK $27.389 \mathrm{U} / \mathrm{L}$ (normal 0-170), lactato-deshidrogenasa (LDH) $710 \mathrm{U} / \mathrm{L}$ (normal 135-250) y glutamato-oxalacetato transaminasa (GOT) $277 \mathrm{U} / \mathrm{L}$. La función renal fue normal y el examen de orina con tira reactiva no reveló hematuria. Fue tratada con hidratación endovenosa con desaparición de las mialgias en 48 horas y descenso de los niveles de CK, sin presentar en ningún momento alteraciones de la función renal u otras complicaciones asociadas. En una revisión posterior a las dos semanas del alta hospitalaria estaba asintomática y las cifras de CK, LDH y GOT fueron normal

Los casos comunicados de rabdomiólisis asociados a EESM se recogen en la tabla 1. El primero fue publicado en 2004, en un paciente que habitualmente no realizaba ejercicio físico y tras un uso repetido del dispositivo (3); en otra observación el cuadro se manifestó tras una sesión única en una paciente no entrenada, en la que el estudio realizado permitió diagnosticar una miopatía metabólica. (4). En el resto, así como en nuestra aportación, la rabdomiólisis se desarrolló tras una única sesión de EESM en individuos que realizaban ejercicio físico regularmente $(5,6)$. Cabe destacar que en uno de los episodios (5) el desencadenante fue la realización de ejercicio y la utilización simultánea de EESM. Todos los casos fueron tratados únicamente con hidratación sin que se observaran complicaciones graves asociada a la rabdomiólisis. En nuestro caso puede descartarse razonablemente la presencia de una miopatía metabólica en ausencia de clínica previa que la sugiera y tras la normalización de las determinaciones de laboratorio durante el seguimiento.

Es previsible que en el futuro aumenten los casos de rabdomiólisis asociados al uso de dispositivos de EESM, dado que su utilización en medicina del deporte 0 en programas de mantenimiento físico, sin supervisión por personal sanitario, es cada vez más amplia. El escaso número de casos comunicados no permite establecer una recomendación que desaconseje por completo su uso, aunque si debería proporcionarse a los usuarios instrucciones para minimizar el riesgo de rabdomiólisis, evitando realizar de forma simultánea ejercicio físico intenso y asegurando una correcta hidratación.
Tabla 1. Casos de rabdomiólisis asociada a electroestimulación muscular comunicados en la literatura.

\begin{tabular}{|l|c|c|c|c|c|c|}
\hline & $\begin{array}{c}\text { Caso 1 } \\
(3)\end{array}$ & $\begin{array}{c}\text { Caso 2 } \\
(4)\left(^{*}\right)\end{array}$ & $\begin{array}{c}\text { Caso 3 } \\
(5)\end{array}$ & $\begin{array}{c}\text { Caso 4 } \\
(6)\end{array}$ & $\begin{array}{c}\text { Caso 5 } \\
(6)\end{array}$ & Caso 6 \\
\hline Edad/Sexo & $26 / N$ & $32 / M$ & $33 / M$ & $19 / N$ & $17 N$ & 25/M \\
\hline Sesión EESM & Repetida & Única & Única & Única & Única & Única \\
\hline $\begin{array}{l}\text { Ejercicio } \\
\text { habitual }\end{array}$ & No & No & Si (**) & Si & Si & Si \\
\hline Pico CK (**) & X10 & X450 & X25 & X700 & X80 & X165 \\
\hline Complicaciones & No & No & No & No & No & No \\
\hline
\end{tabular}

V: varón. M: mujer. EESM: electroestimulación. CK: creatincinasa. El caso 6 es el presentado en este trabajo. ${ }^{(\star)}$ En este caso se diagnosticó además una miopatía metabólica. (*) El cuadro se desencadenó tras realizar simultáneamente EES y ejercicio físico. $\left.{ }^{(\star \star}\right)$ número de veces el límite superior del valor normal.

\section{Referencias}

1. Giannoglou GD, Chatzizisis YS, Misirli G. The syndrome of rhabdomyolysis: Pathophysiology and diagnosis. Eur J Intern Med 2007; 18: 90-100.

2. Bosch X, Poch E, Grau JM. Rhabdomyolysis and acute kidney injury. N Engl J Med 2009; 361: 62-72.

3. Guarascio P, Lusi EA, Soccorsi F. Electronic muscular stimulators: A novel unsuspected cause of rhabdomyolysis. Br J Sports Med 2004; 38: 505.

4. Finsterer J, Stöllberger C. Severe rhabdomyolysis after MIHA-bodytec $®$ electrostimulation with previous mild hyper-CK-emia and noncompaction. Int J Cardiol 2015; 180: $100-2$.

5. Guillén Astete CA, Mondragón S, Medina Quinones C. Rabdomiólisis secundaria a la realización de actividad física y electroestimulación simultánea: reporte de un caso. Reumatol Clin 2015; 11: 262-3.

6. Kästner A, Braun M, Meyer T. Two Cases of Rhabdomyolysis After Training With Electromyostimulation by 2 Young Male Professional Soccer Players. Clin J Sport Med 2015; 25: e71-3

Pablo Ruiz Sada, Lara Palacios García, Itziar Garmendia Antía, Alfonso Gutiérrez Macías.

Servicio de Medicina Interna. Hospital Universitario Basurto. Bilbao. 